\title{
Maximizing botulinum toxin injections for cosmetic and therapeutic applications with a single use, disposable, exact dose injection assist device
}

This article was published in the following Dove Medical Press journal:

Clinical, Cosmetic and Investigational Dermatology

\author{
Marilyn S Kwolek' \\ Jon E Block ${ }^{2}$ \\ 'Private Practice, Danville, CA \\ 94526, USA; ${ }^{2}$ Independent Clinical \\ Consultant, San Francisco, CA 94II5, \\ USA
}

Background: Although freehand injection of botulinum toxin remains the status quo, it is an inaccurate technique for delivering precise amounts of neurotoxin at multiple injection sites with expediency. We developed an inexpensive, ergonomic, single-use, disposable exostructure device that rapidly converts a standard syringe into a highly accurate injection instrument.

Materials and methods: Based on the results of two independent injectors, we determined the comparative delivery dose accuracy, precision, and amount of product waste associated with a standard syringe using the freehand injection technique vs a prototype injection assist device. Fifteen experienced injectors were also surveyed regarding their satisfaction using the device in a controlled research setting.

Results: The average percentage difference from the expected dose value was $<1 \%(0.00024$ $\mathrm{mL}$ ) for 1-unit doses (ie, $0.025 \mathrm{~mL}$ ) with the injection assist device. In contrast, accuracy values with the freehand method were more than 10 times worse (ie, $>10 \%$ or $0.0025 \mathrm{~mL}$ ). Accuracy estimates were also superior with the device over freehand for 2- and 4-unit doses. Precision estimates favored the device for all comparisons and all dosing regimens. The magnitude of variability around the average difference ranged from 2.2 to 5.4 times worse for the freehand method compared to the device. The overall percentage of product waste was substantial with the freehand method ( $>10 \%)$ compared to the device $(4.3 \%)(P=0.04)$ for 1-unit injections. The percentage of injections showing measurable product waste was also significantly lower with the device ( $78 \%$ vs $25 \%, P=0.01$ ). Fourteen of $15(93 \%)$ injectors surveyed would use the device in daily practice if it were included complimentary with the neurotoxin.

Conclusion: The injection assist device offered superior accuracy and precision compared to the freehand method across the entire injection regimen. The ancillary finding of a significant reduction in product waste with the device was particularly notable.

Keywords: neurotoxin, injection, syringe, accuracy

\section{Introduction}

Due in large part to its uniform and undisputed efficacy for cosmetic and therapeutic applications, botulinum toxin has enjoyed ever expanding usage across a broad array of medical practitioners. ${ }^{1}$ Burgeoning demand in patients for nonsurgical cosmetic procedures, in particular, has fueled the ongoing quest for more approved esthetic indications as well as the introduction of newer neurotoxin formulations into the commercial medical marketplace. ${ }^{2}$
Correspondence: Jon E Block 2210 Jackson Street, Ste. 40I, San Francisco, CA 94II5, USA

Tel +l 4I5 7757947

Email jb@drjonblock.com 
Alongside the steep growth trajectory in botulinum toxin utilization has been an evolving understanding of the need to tailor personalized treatment plans to accommodate differing facial anatomies, muscle tone, genders, ethnicities, and patient preferences. ${ }^{3-5}$ In recently published consensus recommendations for esthetic use, Sundaram et $\mathrm{al}^{6}$ described a "paradigm shift" away from the previous emphasis on facial paralysis toward achieving a more relaxed, natural appearance with the elimination of lines in repose while maintaining some movement and facial animation during emotive expression. It is now widely acknowledged that a one-size-fits-all approach to botulinum toxin administration produces less than optimal esthetic results. ${ }^{3}$

In order to consistently realize a more natural appearance in patients, lower doses of neurotoxin distributed over a greater number of injection points are required. ${ }^{1,6-8}$ This technique necessitates that the contents of the syringe should be apportioned reproducibly in separate, discrete doses across multiple injection sites during the same procedure. Although freehand injection of botulinum toxin remains the status quo, it is an inaccurate technique for delivering precise amounts of neurotoxin at multiple injection sites with expediency. In fact, injection dose accuracy with freehand syringe delivery worsens with smaller doses. ${ }^{9}$ Consequently, some practitioners have resorted to using multiple syringes filled with individual units or they pause frequently during the procedure to estimate the remaining contents in the syringe barrel. These methods are burdensome and add unnecessary time and complexity to the procedure.

Given the enormous worldwide clinical demand projected for botulinum toxin, there exists an urgent need to develop improved delivery methods for administering accurate volumetric doses from a standard syringe. We developed an inexpensive, ergonomic, single-use, disposable molded plastic exostructure device that rapidly converts a standard syringe into a highly accurate injection instrument. Herein, we provide comparative quantitative evaluations of the injection assist device accuracy, precision and amount of product waste vs the traditional freehand injection method, as well as a qualitative assessment of user satisfaction with the device.

\section{Materials and methods Overview}

The objective of this study was to determine the comparative delivery dose accuracy, precision, and amount of product waste associated with the use of a standard syringe using the freehand injection technique vs an injection assist device. All quantitative tests were performed by an independent preclinical contract research organization (PMI, San Carlos, CA, USA) using standardized models defined prospectively in specified protocols. This study was exempt from institutional review board review and oversight as all test data were conducted using bench top experiments and did not involve human subjects or live animals.

To evaluate user satisfaction with the injection assist device, we engaged a medical market research firm (Qessential, Exeter, NH, USA) to independently conduct in person, in-depth interviews with experienced, high-volume neurotoxin users in a major US metropolitan center. Each injector experienced "hands on" utilization of the device in a controlled market research setting. Qualitative market research was conducted in accordance with the Market Research Association's Code of Marketing Research Standards (2013, Washington, DC, USA).

\section{Device description}

The injection assist device is an exostructure to assist in the accurate delivery of individual units from a standard, singleuse, disposable syringe filled with multiple doses (KB Medical, LLC, Las Vegas, NV, USA). The syringe exostructure is single-use and disposable and can be configured to support different syringe sizes and dosing requirements. The syringe exostructure includes formed features that ergonomically replicate the impression of a standard syringe (Figure 1). The exostructure retains a syringe in place while a cam mechanism advances the syringe's plunger a set distance into the barrel to expel a measured volume with each compression of the exoskeleton plunger. Tactile and/or audible feedback(s) are provided when the plunger is compressed. The filled syringe is retained within a main body of the syringe exostructure and secured in place by the formed features and a hinged door that closes permanently and is tamper proof. The device specified for this study was configured to deliver individual doses equaling $0.025 \mathrm{~mL}$ with each compression of the plunger.

\section{Accuracy and precision}

The freehand injection protocol was executed by two separate operators on 2 different days. Both operators were experienced injectors with over a decade of neurotoxin injection experience. All injections used normal saline. In all cases, a 1.0-mL standard disposable syringe (B Braun, Bethlehem, PA, USA) with a $30-\mathrm{G}$ needle was used. The dose quantity of saline contained within a $1.0-\mathrm{mL}$ disposable syringe was measured using a digital analytical scale and an individual dose, referred to as 1 "unit", equivalent to a target value of 
A
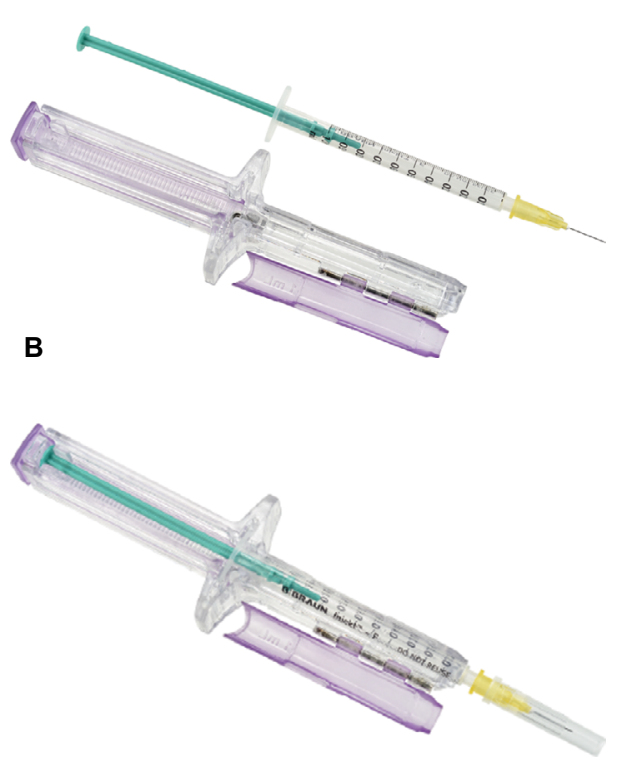

C

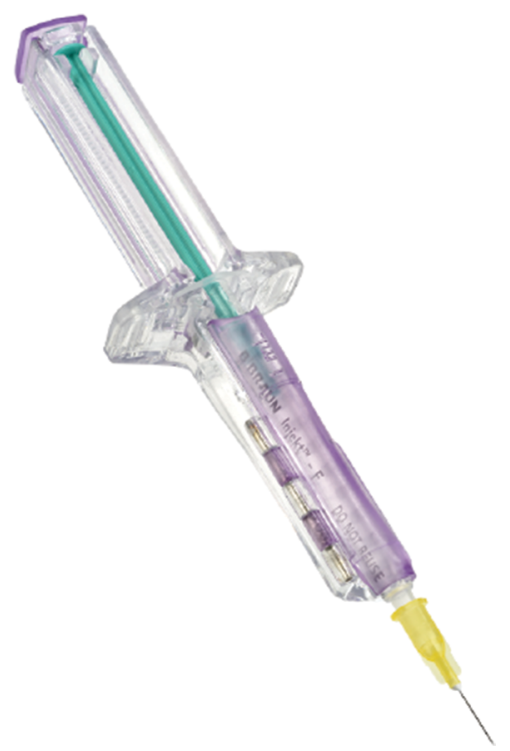

Figure I Injection assist device assembly

Notes: Injection assist device side-by-side with a standard I.0 mL syringe (A). Syringe placed within the injection assist device (B). Syringe secured within the device and fully converted to an accurate injection instrument with tamper-proof hinged door permanently closed and ready to be deployed (C).

$0.025 \mathrm{~mL}$ was delivered in single and multiple units in the following order:

- 1 unit $\times 10$ injections $=10$ units,

- 2 units $\times 5$ injections $=10$ units, and

- 4 units $\times 5$ injections $=20$ units.

Each injection was delivered freehand into an appropriate receptacle atop the analytical scale. The value of the liquid dispensed was recorded after each injection, and the scale was reset prior to the next injection in the sequence.

The two operators were blinded to the measured scale value during the freehand injection process but were allowed visualization of the syringe barrel. The injection regimens were timed and restricted to 3 minutes.

Using the same injection and measurement regimen described earlier, a total of 120 total units of saline were delivered using the injection assist device, reflecting three repetitions of the injection regimen. As before, execution of the injection regimen was timed and limited to 3 minutes per series. Multiunit injections were delivered as consecutive individual units prior to resetting the scale. A single independent operator executed this injection regimen.

\section{Product waste}

Using a turkey leg injection model, the amount of postinjection dripping of saline from the injection site and drips at the tip of the needle were collected using absorbable sponges and measured using the digital analytical scale. Product waste volumes were recorded immediately following needle withdrawal. The same two independent operators were employed to execute the freehand technique product waste protocol.

All injections were delivered into a turkey leg model with skin intact at room temperature. The same injection sequence (ie, 10, 10, and 20 units) was used for this study for both the freehand technique and the injection assist device. For the freehand method, injection sequences involving multiple units (ie, 2 and 4 units) were dispensed as continuous injections. For the injection assist device, multiunit injections were again delivered as consecutive individual units prior to needle withdraw.

\section{User satisfaction}

A qualitative market research approach was used to assess the clinical utility and satisfaction with use of the injection assist among experienced neurotoxin users. The assessment was based on responses to a combination of stimuli (eg, video demonstrations and direct evaluation of actual prototypes of the product). Potential participants were canvassed from a major US metropolitan center.

To qualify, respondents had to fulfill the following:

- Board certified in specialty (physician only).

- Been in practice for at least 1 year and performing neurotoxin cosmetic facial injections for at least 2 years. 
- Must treat at least five patients per week for neurotoxin cosmetic facial injections.

A total of 15 in-depth interviews were conducted among clinicians performing cosmetic facial injections as follows:

- six plastic surgeons,

- seven dermatologists, and

- two extenders (one nurse and one physician's assistant).

On average, interview length was 60 minutes. Although the threshold for inclusion was at least five patients injected per week, 12 of 15 practitioners reported injecting 10 or more patients with 9 of 15 reporting 20 or more patients per week (range: 6-75 per week). All interviews were recorded, transcribed, and qualitatively summarized.

\section{Statistical methods}

All accuracy, precision, and product waste data were managed and analyzed by an independent biostatistical firm (Advanced Research Associates, Santa Clara, CA, USA). Precision and accuracy estimates were computed, summarized, and displayed graphically. The percentage of product waste was also computed and compared across groups.

The accuracy and precision of the freehand and injection assist device methods of delivering product were assessed for 1 unit, 2 units, and 4 units. This assessment was performed by analyzing the differences between the expected and actual volumes delivered. The proximity of the average difference to 0 provides an assessment of the level of accuracy obtained with each method. Precision was assessed by the SD of the differences between the expected and actual volume. SDs and 95\% CIs were plotted for the freehand method and for the device.
During the 4-unit accuracy study, it was noted that the total amount of saline expelled with the injection assist device was $<1.0 \mathrm{~mL}$ for all three repetitions. This resulted in the final 1 -unit dose in the 4-unit dose sequence being substantially $<0.025 \mathrm{~mL}$ in each case. Thus, results are presented with the final 4-unit dose removed from the analysis.

Statistical comparisons between techniques used the twosample, $t$-test (two-tailed) for accuracy and product waste estimates and the F-test to compare the SDs as an estimate of precision. Bland-Altman plots were constructed to determine the degree of agreement between accuracy values achieved with the injection assist device and the expected value (ie, $0.025 \mathrm{~mL} / \mathrm{unit}$ ). ${ }^{10}$ The Bland-Altman method calculates the mean difference between two methods of measurement (the "bias") and the $95 \%$ limits of agreement as the mean difference (1.96 SD).

\section{Results}

There was a substantial improvement in injection accuracy and precision with the injection assist device compared to the traditional freehand method. Tables $1-3$ provide accuracy and precision summary statistics for the injection assist device as well as for both freehand operators separately and combined. For accuracy, the average percentage difference from the expected dose value was $<1 \%(0.00024 \mathrm{~mL})$ for 1 -unit doses (ie, $0.025 \mathrm{~mL}$ ) with the injection assist device. In contrast, both operators had accuracy values that were more than 10 times worse than the device (ie, $>10 \%$ or $0.0025 \mathrm{~mL})(P=0.09$ vs operator $1 ; P=0.24$ vs operator 2$)$. Similar differences in favor of the device were found for 2- and 4-unit doses (Tables 2 and 3). Device accuracy was significantly better

Table I Accuracy and precision summary statistics: I-unit injections

\begin{tabular}{|l|l|l|l|l|l|l|l|}
\hline & N & Mean & SD & Minimum & Maximum & Range & $\begin{array}{l}\text { Relative difference, } \\
\%(\mathbf{m e a n} / \mathbf{0 . 0 2 5})\end{array}$ \\
\hline Freehand operator I & 10 & 0.0026 & 0.003806 & -0.006 & 0.007 & 0.013 & 10.40 \\
\hline Freehand operator 2 & 10 & -0.0028 & 0.007613 & -0.02 & 0.008 & 0.028 & -11.20 \\
\hline Freehand combined & 20 & -0.0001 & 0.00648 & -0.02 & 0.0080 & 0.028 & -0.40 \\
\hline Device & 29 & 0.00024 & 0.001725 & -0.004 & 0.003 & 0.007 & 0.97 \\
\hline
\end{tabular}

Table 2 Accuracy and precision summary statistics: 2-unit injections

\begin{tabular}{|l|l|l|l|l|l|l|l|}
\hline & $\mathbf{N}$ & Mean & SD & Minimum & Maximum & Range & $\begin{array}{l}\text { Relative difference, } \\
\%(\mathbf{m e a n} / \mathbf{0 . 0 5})\end{array}$ \\
\hline Freehand operator I & 5 & 0.01 & 0.003162 & -0.015 & -0.007 & 0.008 & 20.00 \\
\hline Freehand operator 2 & 5 & 0.0022 & 0.004382 & -0.01 & 0 & 0.01 & 4.40 \\
\hline Freehand combined & 10 & 0.0061 & 0.005466 & -0.015 & 0.00000 & 0.015 & 12.20 \\
\hline Device & 15 & -0.00067 & 0.000816 & -0.002 & 0 & 0.002 & -1.33 \\
\hline
\end{tabular}


than that achieved by operator 1 for 2 -unit $(P=0.001)$ and 4-unit $(P<0.0001)$ injections, but not for operator $2(P \geq 0.11$ for both comparisons). In particular, it was notable that the accuracy estimate was only $0.5 \%$ for 4 -unit doses with the device (Table 3), reflecting excellent accuracy.

Comparative accuracy estimates and their associated variability are also displayed graphically (Figures 2-4). Inspection of these figures illustrates not only the relative proximity to " 0 " achieved with the device, reflecting excellent accuracy for all dosing regimens, but also the wide variability in accuracy between the 2 independent operators using the freehand method.
Precision estimates also favored the device for all comparisons and all dosing regimens (Tables $1-3$ ). The magnitude of variability around the average difference ranged from 2.2 to 5.4 times worse for the freehand method compared to the injection assist device. Precision estimates were significantly better for the device compared to the combined freehand values for all 3 dosing regimens $(P<0.001$ for all comparisons). The substantial differences in precision between the device and the freehand method are most notable graphically (Figures 5-7).

Bland-Altman plots are provided to display the agreement between the expected and delivered volumes for the injection

Table 3 Accuracy and precision summary statistics: 4-unit injections

\begin{tabular}{|l|l|l|l|l|l|l|l|}
\hline & $\mathbf{N}$ & Mean & SD & Minimum & Maximum & Range & $\begin{array}{l}\text { Relative difference, } \\
\%(\mathbf{m e a n} / \mathbf{0 . 1})\end{array}$ \\
\hline Freehand operator I & 5 & 0.0472 & 0.005357 & 0.038 & 0.052 & 0.014 & 47.20 \\
\hline Freehand operator 2 & 5 & -0.0032 & 0.004087 & -0.008 & 0.001 & 0.009 & -3.20 \\
\hline Freehand combined & 10 & 0.0220 & 0.02694 & -0.008 & 0.052 & 0.06 & 22.00 \\
\hline Device & 12 & 0.0005 & 0.001 & -0.001 & 0.002 & 0.003 & 0.50 \\
\hline
\end{tabular}

\subsection{8}

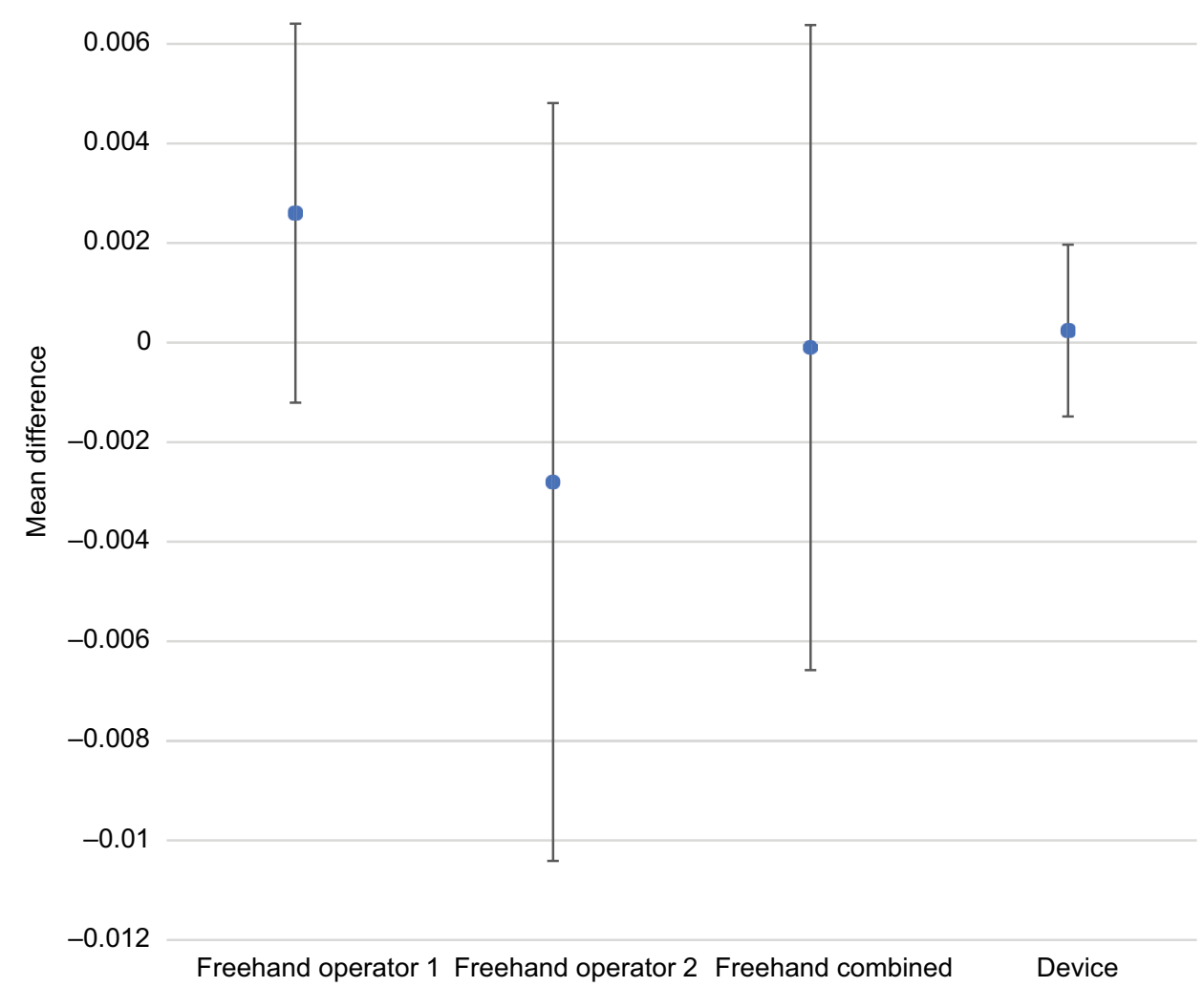

Figure 2 Comparative accuracy estimates for I-unit $(0.025 \mathrm{~mL})$ doses.

Note: Data are expressed as mean difference $\pm I S D$. 


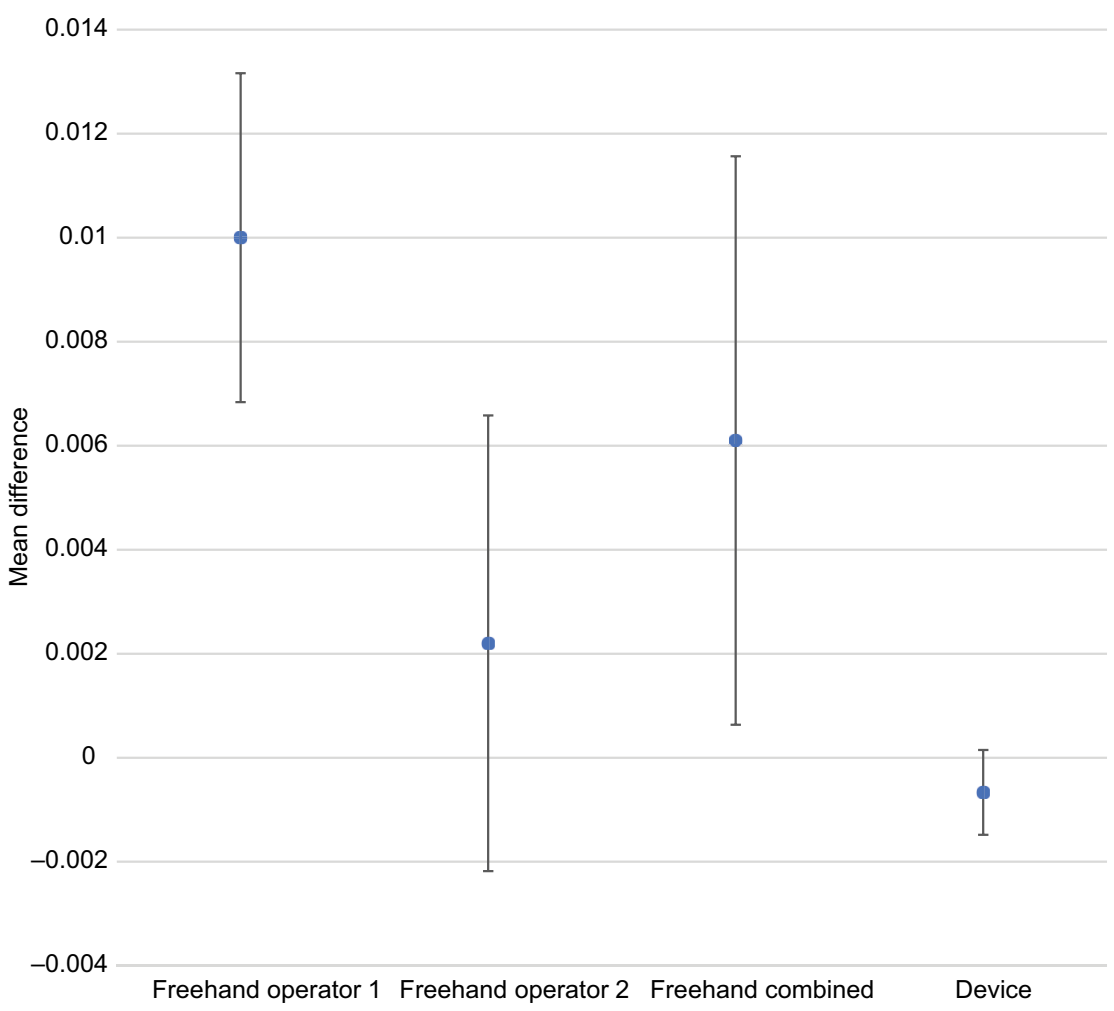

Figure 3 Comparative accuracy estimates for 2-unit $(0.05 \mathrm{~mL})$ doses.

Note: Data are expressed as mean difference \pm I SD.

0.06

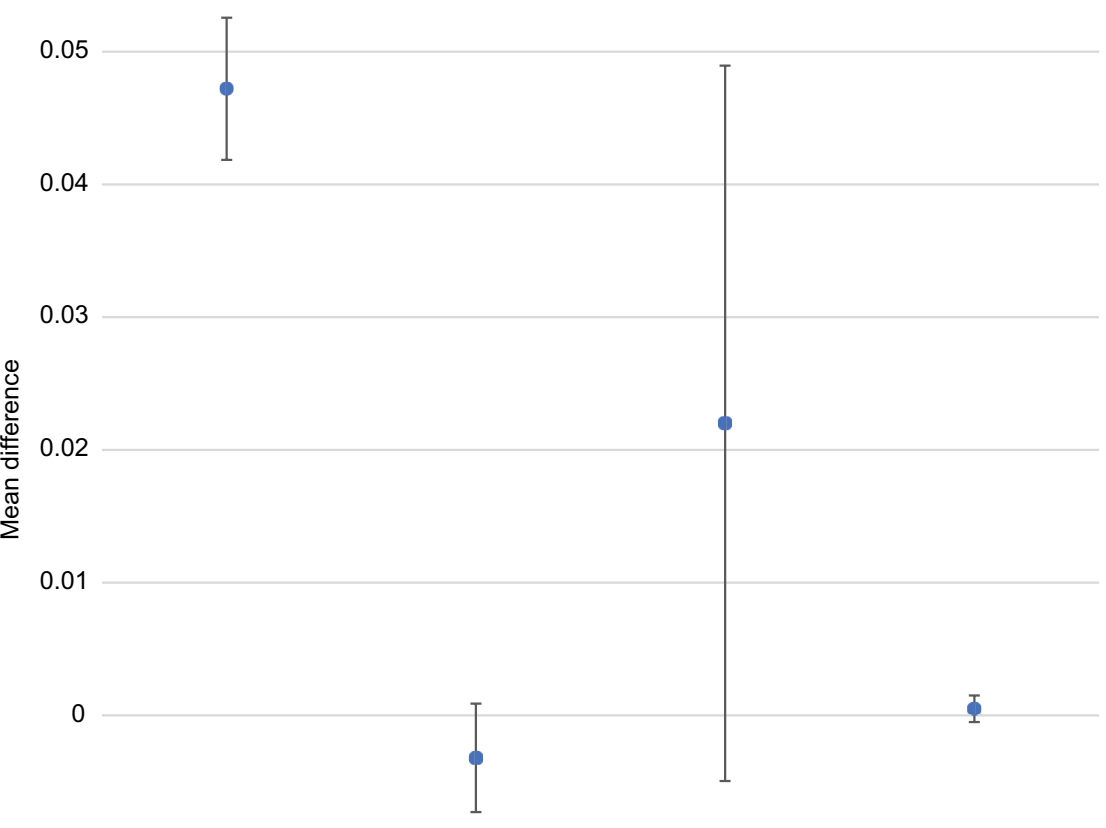

$-0.01$

$-0.02$

Freehand operator 1 Freehand operator 2 Freehand combined Device

Figure 4 Comparative accuracy estimates for 4 -unit $(0.10 \mathrm{~mL})$ doses.

Note: Data are expressed as mean difference $\pm I$ SD. 
0.016

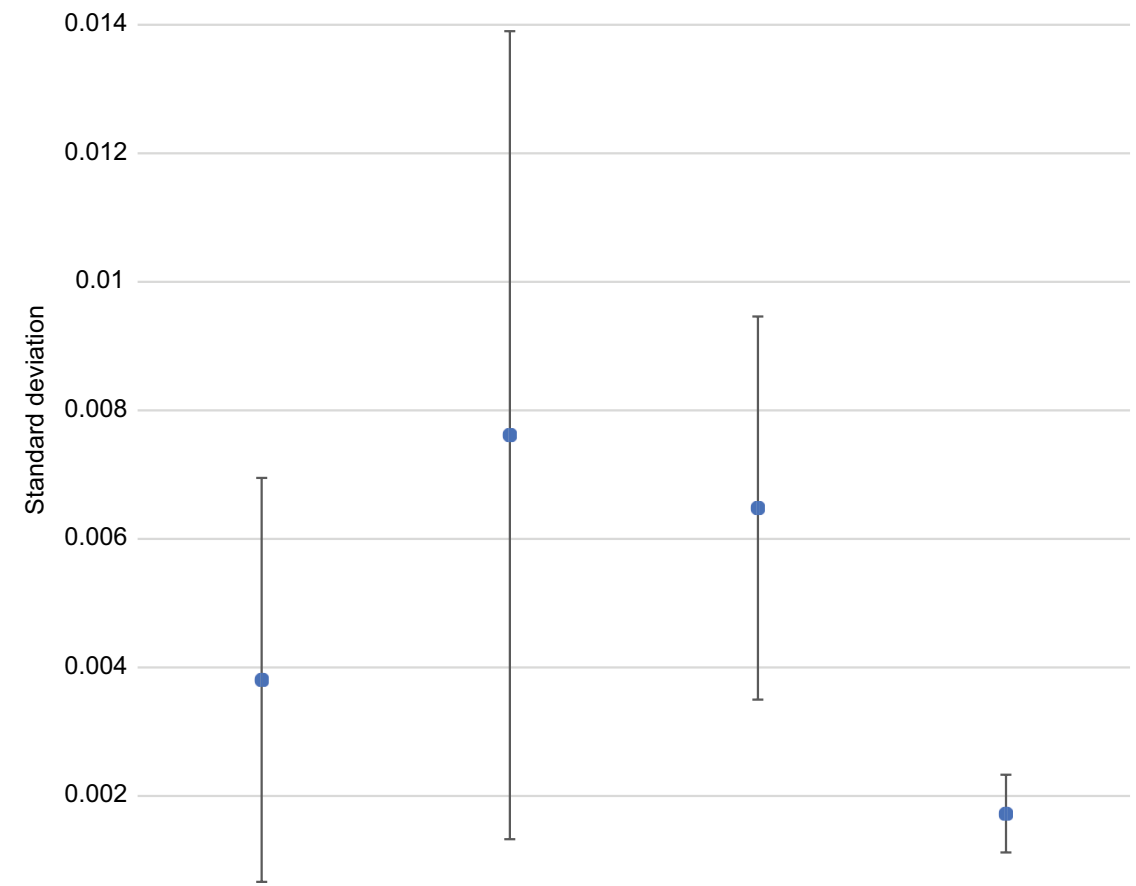

0

Freehand operator 1 Freehand operator 2 Freehand combined Device

Figure 5 Comparative precision estimates for I-unit $(0.025 \mathrm{~mL})$ doses.

Note: Data are expressed as SD with $95 \%$ Cls.

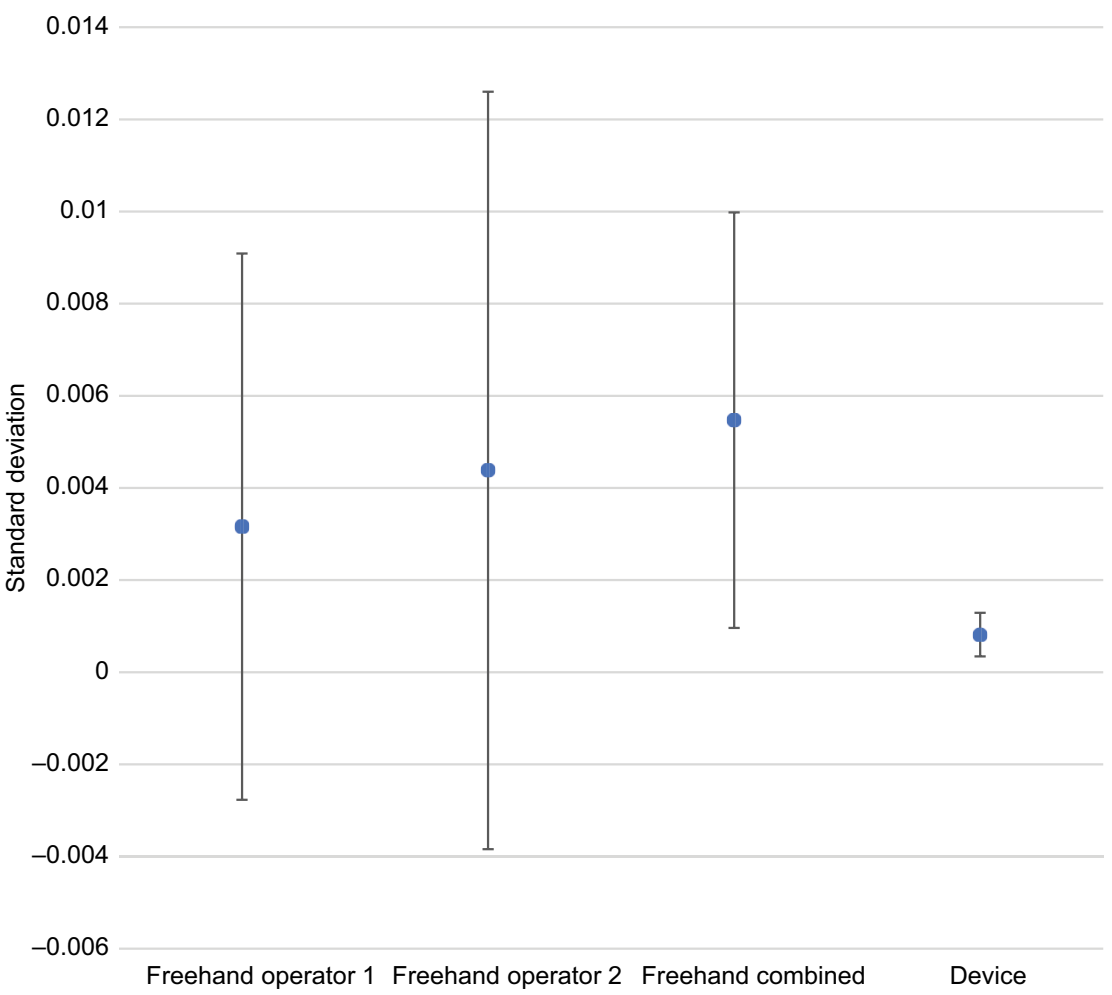

Figure 6 Comparative precision estimates for 2-unit $(0.05 \mathrm{~mL})$ doses.

Note: Data are expressed as SD with $95 \%$ Cls. 
0.06

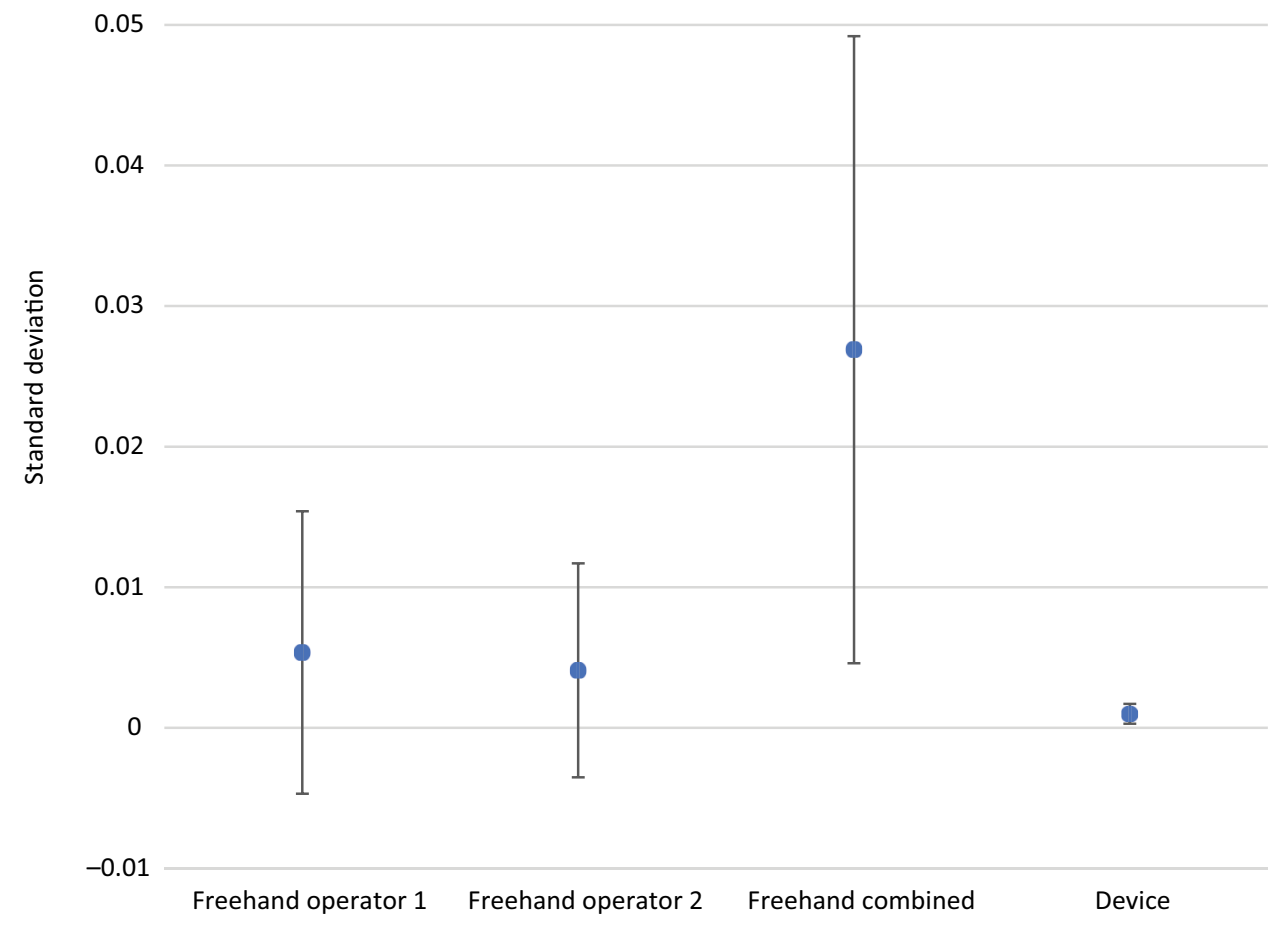

Figure 7 Comparative precision estimates for 4-unit $(0.10 \mathrm{~mL})$ doses.

Note: Data are expressed as SD with $95 \% \mathrm{Cls}$.

assist device, with the $\mathrm{X}$-axis representing the order of injection (Figures 8-10). There was excellent agreement between the amount delivered and the expected value (ie, $0.025 \mathrm{~mL} /$ unit) with mean differences of $0.0002,0.0007$, and 0.0005 $\mathrm{mL}$ for 1-, 2-, and 4-unit injections, respectively.

The percentage of product waste relative to the total amount dispensed was calculated per injection for each method (Tables 4-6). A substantial reduction in product waste was realized with the injection assist device compared to the freehand method. For example, for 1-unit injections, the percentage of product waste was $11.9 \%$ for the mean combined total of the 2 independent operators. This value was 2.8 times higher than the average percentage of product waste associated with the device (4.3\%) $(P=0.04)$. For 4-unit doses, the average percentage of product waste was 6.2 times higher ( $3.1 \%$ vs $0.5 \%, P=0.13$ ) for freehand compared to device. Of note, $78 \%$ (31 of 40) of the freehand injections (both operators combined) resulted in some amount of measurable product waste. In contrast, with the device only $25 \%$ (5 of 20) of the injections showed waste $(P=0.01)$.

In terms of user satisfaction and clinical utility, based on direct hands-on use of the prototype, $87 \%$ (13 of 15) of the injectors surveyed agreed to try the device in daily practice if it were commercially available, and 14 of the 15 (93\%) injectors surveyed would use the device in daily practice if it were included complimentary with the neurotoxin.

\section{Discussion}

This study demonstrated excellent accuracy and precision with the injection assist device that was consistently superior to the freehand method of injection. For example, the relative percentage difference from the expected dose value was $\sim 1 \%$ for 1 - and 2 -unit injections and $<1 \%$ for 4-unit injections with the injection assist device. In sharp contrast, freehand injection accuracy was often greater than 10 times worse (ie, $>10 \%$ inaccuracy). Precision estimates also favored the device by a large degree, resulting in substantially improved consistency and reproducibility between injections compared with the traditional freehand injection method. Consequently, the injection assist device can be employed to instantaneously convert a standard syringe into a highly accurate and precise injection instrument without additional complexity or measurable costs to the procedure. 


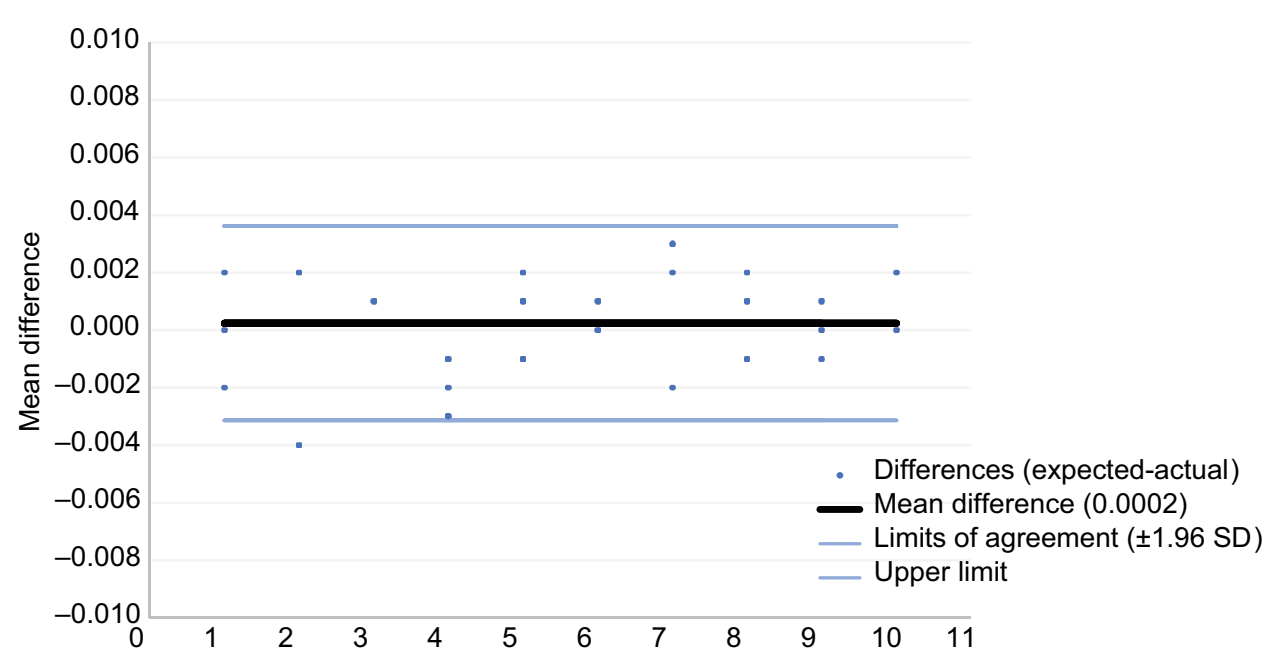

Figure 8 Bland-Altman plot for I-unit $(0.025 \mathrm{~mL})$ doses with $95 \%$ limits of agreement. Note: $\mathrm{X}$-axis shows order of injection.

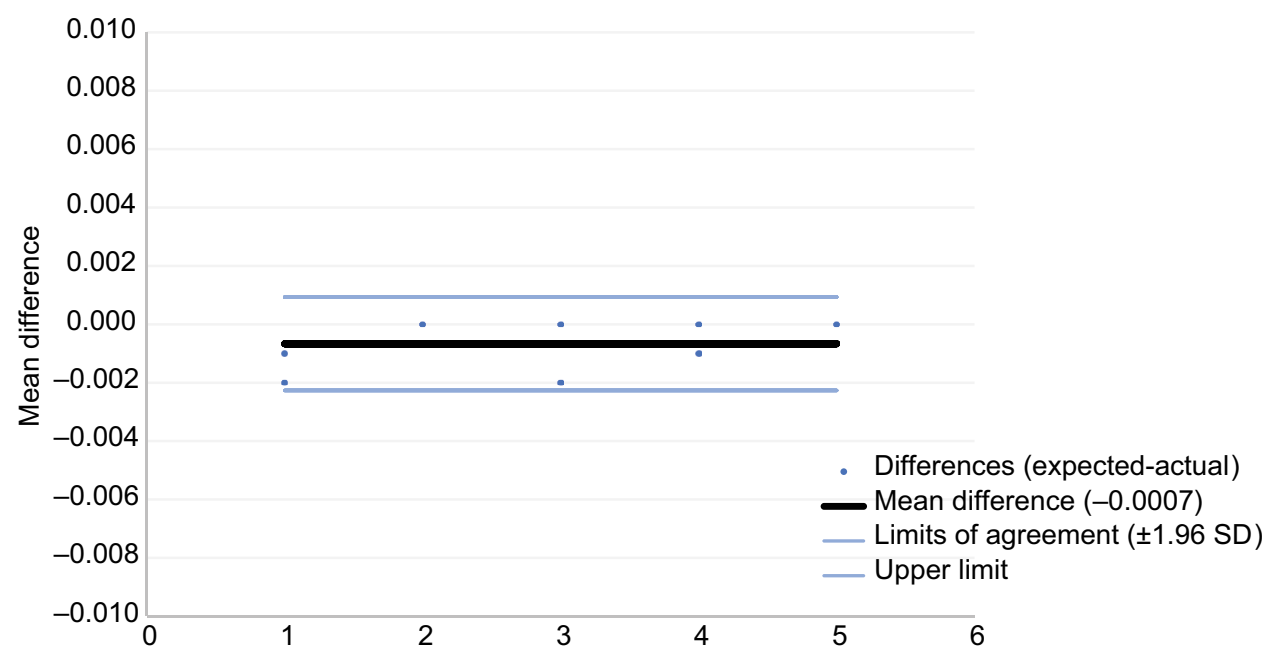

Figure 9 Bland-Altman plot for 2-unit $(0.05 \mathrm{~mL})$ doses with $95 \%$ limits of agreement. Note: $X$-axis shows order of injection.

Product waste during the botulinum toxin injection procedure is a begrudgingly accepted and universal fact of life even for experienced injectors, as confirmed in this study. The product waste comparisons were particularly noteworthy. For 1 -unit doses, percentage product waste exceeded $10 \%$ for both operators, demonstrating a substantial loss of product. In sharp contrast, product waste was $\sim 4 \%$ with the device for 1-unit doses. This is a specifically salient finding considering the current embrace of lower dose, multi-injection regimens to accommodate individualized treatment plans. ${ }^{3,4}$ The undesirable economic consequences of neurotoxin product waste have been documented previously with regard to the selection of syringe type, ${ }^{11}$ but this is the first study to quantify the amount of product waste associated with needle and injection site drips. The ramifications of product waste will also likely be magnified by the shift toward other smaller dose applications such as rejuvenation of facial skin quality requiring numerous microdose injections throughout the lower face. ${ }^{12}$

We hypothesize that the much higher injection rate (dose/ second) capability with our device provides maximum neurotoxin delivery and tissue dispersion, minimizing product waste from injection sites and needle dripping.

A substantial portion of the projected growth in cosmetic neurotoxin use will involve injection by physician extenders in the medical spa setting. ${ }^{13,14}$ It is currently estimated that $\geq 30 \%$ 


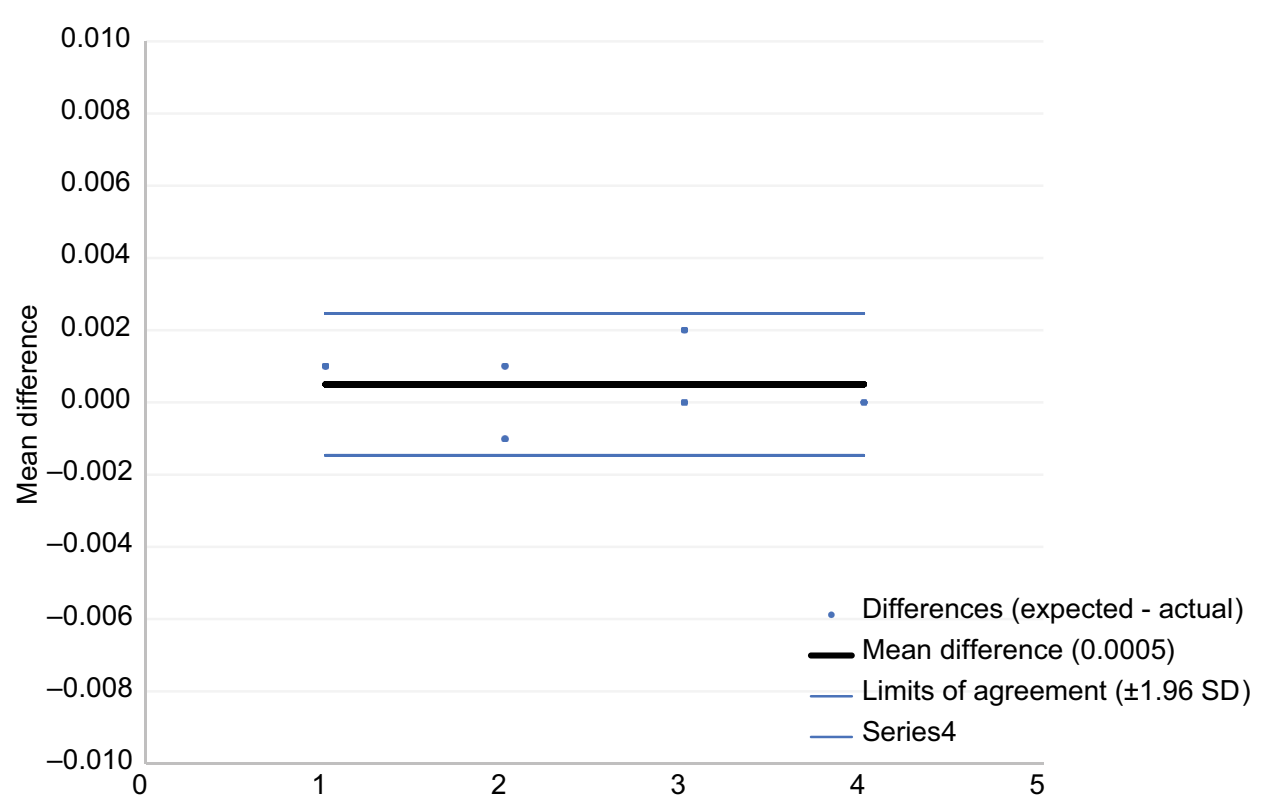

Figure 10 Bland-Altman plot for 4-unit $(0.10 \mathrm{~mL})$ doses with $95 \%$ limits of agreement.

Note: $X$-axis shows order of injection.

Table 4 Comparative percentage product waste: I-unit injections

\begin{tabular}{|l|l|l|l|l|l|}
\hline & N & Mean (\%) & SD (\%) & Minimum (\%) & Maximum (\%) \\
\hline Freehand operator I & 10 & 12.5 & 9.0 & 0 & 27.3 \\
\hline Freehand operator 2 & 10 & 11.2 & 9.1 & 0 & 25.0 \\
\hline Freehand combined & 20 & 11.9 & 8.8 & 0 & 27.3 \\
\hline Device & 10 & 4.3 & 10.0 & 0 & 30.4 \\
\hline
\end{tabular}

Table 5 Comparative percentage product waste: 2-unit injections

\begin{tabular}{|l|l|l|l|l|l|}
\hline & N & Mean (\%) & SD (\%) & Minimum (\%) & Maximum (\%) \\
\hline Freehand operator I & 5 & 6.0 & 2.8 & 2.0 & 10.0 \\
\hline Freehand operator 2 & 5 & 3.1 & 3.2 & 0 & 7.5 \\
\hline Freehand combined & 10 & 4.6 & 3.2 & 0 & 10.0 \\
\hline Device & 5 & 2.0 & 2.8 & 0 & 6.0 \\
\hline
\end{tabular}

Table 6 Comparative percentage product waste: 4-unit injections

\begin{tabular}{|l|l|l|l|l|l|}
\hline & N & Mean (\%) & SD (\%) & Minimum (\%) & Maximum (\%) \\
\hline Freehand operator I & 5 & 5.4 & 2.4 & 2.0 & 8.0 \\
\hline Freehand operator 2 & 5 & 0.8 & 1.1 & 0 & 2.0 \\
\hline Freehand combined & 10 & 3.1 & 3.0 & 0 & 8.0 \\
\hline Device & 5 & 0.5 & 1.0 & 0 & 2.1 \\
\hline
\end{tabular}

of the cosmetic botulinum toxin procedures are performed by physician extenders, often in satellite medical spa facilities under the auspices of a physician director (https://www. surgery.org/sites/default/files/ASAPS-Stats2016.pdf, $\underline{\text { https:// }}$ www.surgery.org/sites/default/files/ASAPS-Stats2017.pdf).
This segment is growing rapidly and would benefit greatly from a device that guaranteed accurate and precise neurotoxin dose delivery and minimized product waste.

With the injection assist device, the injector does not have to rely on visually monitoring the marks on the syringe or 
survey the blebs at the injection site to judge the quantity being injected. The fact that the device allows for the practitioner to be more "patient-focused" was a clear advantage that resonated with injectors in our qualitative assessment of user satisfaction. Indeed, all but one experienced practitioner we surveyed were eager to use the device in clinical practice if it were provided complimentary with the neurotoxin.

Working and feeling like a standard syringe, each time the thumb presses the plunger a single exact unit of neurotoxin is delivered to the injection site. Tactile feedback and a soft audible "click" are provided as valuable features to assist the user in rapid injection. Importantly, the device can easily be configured to accommodate different syringe sizes and dosing requirements.

This device provides a substantially greater level of accuracy and precision compared to the freehand injection technique. Indeed, there are specialty syringe devices on the market that also provide accurate volumetric fluid delivery. ${ }^{15-17}$ However, they are expensive and not single-use or ergonomic. Instead, practitioners have grown accustomed to using inexpensive, off-the-shelf, disposable syringes to deliver botulinum toxin for cosmetic purposes. There is limited interest in transitioning to expensive specialty syringes to achieve the benefit of better injection accuracy. Our device, in contrast, combines the familiarity of using standard disposable syringes with vastly improved injection accuracy and precision in an easy to use format.

\section{Conclusion}

The injection assist device offered superior accuracy and precision compared to the freehand method across the entire injection regimen. The ancillary finding of a significant reduction in product waste with the device was particularly notable. Potential users encouraged its use and expressed interest in employing this device in daily practice.

\section{Acknowledgment}

In this study, graphical support was provided by Terry Meredith.

\section{Author contributions}

Both authors contributed to data analysis, drafting or revising the article, gave final approval of the version to be published, and agree to be accountable for all aspects of the work.

\section{Disclosure}

MSK and JEB are co-founders of KB Medical, LLC (Las Vegas, NV, USA). The authors report no other conflicts of interest in this work.

\section{References}

1. Lanoue J, Dong J, Do T, Goldenberg G. An update on neurotoxin products and administration methods. Cutis. 2016;98(3):163-166.

2. Carruthers J, Fournier N, Kerscher M, Ruiz-Avila J, Trindade de Almeida AR, Kaeuper G. The convergence of medicine and neurotoxins: a focus on botulinum toxin type $\mathrm{A}$ and its application in aesthetic medicine - a global, evidence-based botulinum toxin consensus education initiative: part II: incorporating botulinum toxin into aesthetic clinical practice. Dermatol Surg. 2013;39(3 Pt 2):510-525.

3. Anido J, Arenas D, Arruabarrena C, et al. Tailored botulinum toxin type A injections in aesthetic medicine: consensus panel recommendations for treating the forehead based on individual facial anatomy and muscle tone. Clin Cosmet Investig Dermatol. 2017;10:413-421.

4. Ahn BK, Kim YS, Kim HJ, Rho NK, Kim HS. Consensus recommendations on the aesthetic usage of botulinum toxin type A in Asians. Dermatol Surg. 2013;39(12):1843-1860.

5. Keaney TC, Alster TS. Botulinum toxin in men: review of relevant anatomy and clinical trial data. Dermatol Surg. 2013;39(10): 1434-1443.

6. Sundaram H, Signorini M, Liew S, et al. Global aesthetics consensus: botulinum toxin type $\mathrm{A}$ - evidence-based review, emerging concepts, and consensus recommendations for aesthetic use, including updates on complications. Plast Reconstr Surg. 2016;137(3):518e-529e.

7. Iozzo I, Tengattini V, Antonucci VA. Multipoint and multilevel injection technique of botulinum toxin A in facial aesthetics. J Cosmet Dermatol. 2014;13(2):135-142.

8. Steinsapir KD, Rootman D, Wulc A, Hwang C. Cosmetic microdroplet botulinum toxin A forehead lift: a new treatment paradigm. Ophthalmic Plast Reconstr Surg. 2015;31(4):263-268.

9. Thobani SU, Steward DJ. The accuracy and variability of bolus injections with different sized syringes. Can J Anaesth. 1992;39(2):198-201.

10. Bland JM, Altman DG. Statistical methods for assessing agreement between two methods of clinical measurement. Lancet. 1986;1(8476):307-310.

11. Foglietti MA, Wright L, Foglietti-Fostyk A. Botulinum toxin therapy: is syringe type related to cost-effectiveness? Ann Plast Surg. 2018;80(3):287-289.

12. Zhu J, Ji X, Xu Y, et al. The efficacy of intradermal injection of type A botulinum toxin for facial rejuvenation. Dermatol Ther. 2017;30(1):e12433.

13. Goldman MP. Technology approaches to the medical spa: art plus science equal rejuvenation. Dermatol Clin. 2008;26(3):327-340.

14. Matheson-Shedrick K. Spas unwrapped. J Med Pract Manage. 2008;24(3):166-169.

15. Bertossi D, Mortellaro C, Nocini P. New clinical analysis and device for botox injections. J Craniofac Surg. 2016;27(6):1554-1557.

16. Fukamizu H, Fujiwara M, Kim T, Matsushita Y, Tokura Y. Development of a three-microneedle device for hypodermic drug delivery and clinical application. Plast Reconstr Surg. 2012;130(2):451-455.

17. Torrisi BM, Zarnitsyn V, Prausnitz MR, et al. Pocketed microneedles for rapid delivery of a liquid-state botulinum toxin A formulation into human skin. J Control Release. 2013;165(2):146-152. 


\section{Publish your work in this journal}

Clinical, Cosmetic and Investigational Dermatology is an international, peer-reviewed, open access, online journal that focuses on the latest clinical and experimental research in all aspects of skin disease and cosmetic interventions. This journal is included on PubMed. The manuscript management system is completely online

Submit your manuscript here: https://www.dovepress.com/clinical-cosmetic-and-investigational-dermatology-journal

and includes a very quick and fair peer-review system, which is all easy to use. Visit http://www.dovepress.com/testimonials.php to read real quotes from published authors 